\title{
Minería, formas de urbanización y transformación del espacio en Huamachuco, La Libertad, Perú
}

Exploitation minière, types d'urbanisation et transformation de l'espace à Huamachuco, La Libertad, Pérou

Mining, forms of urbanization and space transformation in Huamachuco, La Libertad, Peru

\section{Alejandro Gonzales Gavilano}

\section{OpenEdition}

\section{Journals}

Edición electrónica

URL: http://journals.openedition.org/bifea/8968

DOI: $10.4000 /$ bifea.8968

ISSN: 2076-5827

\section{Editor}

Institut Français d'Études Andines

\section{Edición impresa}

Fecha de publicación: 8 diciembre 2017

Paginación: 509-527

ISSN: 0303-7495

\section{Referencia electrónica}

Alejandro Gonzales Gavilano, « Minería, formas de urbanización y transformación del espacio en Huamachuco, La Libertad, Perú », Bulletin de l'Institut français d'études andines [En línea], 46 (3) | 2017 Publicado el 08 diciembre 2017, consultado el 05 noviembre 2020. URL : http:// journals.openedition.org/bifea/8968; DOI : https://doi.org/10.4000/bifea.8968

Les contenus du Bulletin de l'Institut français d'études andines sont mis à disposition selon les termes de la licence Creative Commons Attribution - Pas d'Utilisation Commerciale - Pas de Modification 4.0 International. 


\title{
Minería, formas de urbanización y transformación del espacio en Huamachuco, La Libertad, Perú
}

\author{
Alejandro Gonzales Gavilano*
}

\begin{abstract}
Resumen
La minería juega un papel importante en la estructuración del territorio peruano. Sin embargo, la minería es heterogénea: existen distintas escalas y formas de operación que generan distintas formas de relación con el territorio y el sistema urbano. Este artículo busca explorar los cambios que se dan en los centros urbanos que son influenciados por proyectos mineros. En primer lugar, se hace un recuento de las problemáticas que vinculan la minería con la urbanización. En segundo lugar, se explican ciertos territorios y centros mineros en el Perú. Finalmente, se expone el caso de la ciudad de Huamachuco, capital de provincia en los Andes del norte del Perú, y los efectos urbanos que pueden tener cuatro operaciones mineras actualment operativas en la zona.
\end{abstract}

Palabras clave: minería, urbanización, Perú, Huamachuco

\section{Exploitation minière, types d'urbanisation et transformation de I'espace à Huamachuco, La Libertad, Pérou}

\section{Résumé}

Les exploitations minières jouent un rôle important dans la structuration du territoire péruvien. Cependant, les activités minières sont hétérogènes : il y a plusieurs échelles et formes d'exploitation qui génèrent différents types de liens avec le territoire et le système urbain. Cet article cherche à explorer les changements que subissent les centres urbains qui sont sous l'influence de projets miniers. Tout d'abord, sont exposées les problématiques autour des liens entre exploitations minières et urbanisation. Ensuite, sont décrits certains territoires et centres miniers au Pérou. Finalement, l'auteur expose le cas de la ville de Huamachuco, capitale de province dans les Andes nord du Pérou, et les effets sur l'urbanisme issus de la présence de quatre mines proches de la ville.

* Pontificia Universidad Católica del Perú, Av. Universitaria, 1801, San Miguel. E-mail: agonzalesg@ pucp.pe 
Mots-clés : exploitations minières, urbanisation, Pérou, Huamachuco

\title{
Mining, forms of urbanization and space transformation in Huamachuco, La Libertad, Peru
}

\begin{abstract}
Mining plays an important role in the structuring of the Peruvian territory. However, mining activities are heterogeneous: there are different scales and types of mining operations that generate different kinds of linkages with the territory and the urban system. Our aim is to explore the changes that occur in urban centers that are influenced by mining operations. Firstly, we discuss the links between mining and urbanization. Secondly, we describe certain territories and mining centers in Peru. Finally we present the case of the city of Huamachuco, a provincial capital in Peru's northern Andes, that is under the influence of four mining operations.
\end{abstract}

Key words: mining, urbanization, Peru, Huamachuco

\section{INTRODUCCIÓN}

Hoy el mundo se encuentra hacia el final del llamado «súper ciclo» minero, en que los precios y la demanda por minerales aumentaron en gran medida. Este súper-ciclo, nombre dado a un periodo en que la demanda por materias primas es regularmente alta, se ha dado durante la década de los años 2000 y se explica en buena medida por un incremento de la demanda de la industria asiática. En este contexto, en el Perú, se han multiplicado los proyectos mineros en el territorio, algunos en zonas mineras ya conocidas, y otros en zonas en que históricamente la minería ha sido menos intensa, pero con reservas minerales importantes. Esto ha dado lugar a múltiples procesos de cambio en el territorio: expansión urbana de ciudades cercanas a minas, creación de nuevos enclaves, reasentamientos involuntarios, creación de nuevos centros poblados, declive de ciertas ciudades, etc. De igual manera, en el contexto de los precios altos de los commodities, las explotaciones mineras informales de menor escala se han activado en muchos lugares del territorio peruano, dando lugar a nuevos centros o dando nueva vida a otros ya existentes.

Actualmente, el debate nacional alrededor del papel que juega la minería en el desarrollo del país suele ubicarse entre dos posiciones casi antagónicas: una primera que preconiza las ventajas y beneficios de la minería para la economía nacional, y una segunda que pone en evidencia el daño ambiental y las vulneraciones a los derechos de los ciudadanos que se ven afectados por proyectos mineros y se opone a la actividad. Estas dos posiciones, aquí enunciadas de manera muy simple, parecen tener dificultad en encontrar puntos en común que podrían abrir caminos para solucionar las aparentes contradicciones. Los instrumentos, procesos y planes de ordenamiento territorial se han evidenciado como necesarios, sin embargo se encuentran aun en estados iniciales. 
Múltiples estudios se han llevado a cabo sobre los impactos de la minería: estudios sobre impactos en el medio ambiente, en los espacios rurales, en la formación de conflictos sociales, en las dinámicas económicas. Sin embargo, los vínculos entre minería y urbanización son un campo aún poco explorado. Las ciudades son espacios estructuradores del territorio que hoy se relacionan con unidades y proyectos mineros. En el contexto de urbanización planetaria en que cada vez más personas habitan en ciudades, el rol de las ciudades secundarias e intermedias se revela importante para el desarrollo. Más aún en el Perú, en que la mayor parte de las minas se encuentra en la región andina, espacio articulado por ciudades de este tipo.

Nos preguntamos de qué manera los nuevos proyectos mineros se han relacionado con las ciudades o los centros urbanos y qué tipos de vínculos existen entre la minería y los procesos de urbanización y de producción del espacio.

Nos centramos en el caso de la ciudad de Huamachuco, capital de la provincia de Sánchez Carrión, región de La Libertad. Se trata de una ciudad secundaria del sistema urbano en los Andes del norte del país que se encuentra relacionada con varias operaciones mineras de distintas naturalezas y escalas de explotación. Sostenemos que Huamachuco, de ser un centro articulador de un espacio más bien rural, se ha vuelto funcional a ciertas necesidades de unidades mineras que se encuentran próximas a esta ciudad, lo cual se traduce en cambios en la morfología urbana y en el desarrollo de redes territoriales.

\section{LOS VÍNCULOS ENTRE MINERÍA, URBANIZACIÓN Y DESARROLLO}

\section{1. Minería y escala de análisis}

El proceso urbano ya no se puede entender únicamente observando a cada ciudad de manera aislada. Todo proceso de urbanización tiene una correlación con otros espacios $u$ otras ciudades con las que establece relaciones de distinto tipo. Es decir que un análisis urbano requiere de una mirada más amplia que abarque al territorio y al sistema urbano al cual cada ciudad pertenece. Esto es particularmente importante cuando se habla de industrias extractivas hoy, pues el mercado de las materias primas está compuesto por flujos a escala del mundo. De igual forma, las minas suelen encontrarse en lugares alejados de centros urbanos, poco accesibles y de geografía difícil. Por lo tanto, la relación entre la minería y la ciudad abarca relaciones que van más allá de la influencia de una mina en una ciudad, sino que implica un grado de influencia en espacios mayores del territorio. En este sentido, Bryceson \& MacKinnon (2012) proponen el concepto de «urbanización mineralizada» que definen como la «influencia de los ciclos de producción mineral y de las cadenas de mercancías (commodities) sobre el crecimiento urbano y los patrones de establecimiento a nivel local, regional y nacional». Es decir que el impacto de la actividad minera se da a múltiples escalas del territorio y que los proyectos mineros pueden inducir reconfiguraciones 
espaciales a varios niveles. Estas reconfiguraciones tienen que ver con procesos migratorios desencadenados por la atracción que puede generar la actividad económica, ya sea directamente o indirectamente, ligada a la minería (servicios o comercio, por ejemplo).

El análisis que se haga deberá abarcar por lo menos dos escalas, una ligada al territorio inmediato de una mina y su área de influencia (incluyendo los centros urbanos directamente ligados), y otra que mire el sistema urbano más amplio en el que se inserta dicha mina.

\section{2. Centros urbanos mineros}

En este caso, se prefiere emplear el término centro urbano (minero) en vez de ciudad (minera). El término de «ciudad» se suele asociar más a un espacio de un cierto tamaño que reúne algunas características tales como albergar una especialización del trabajo, tener jerarquías sociales, mantener una relación estrecha con su entorno rural y distinguirse por alguna definición monumental (Kostof, 1991). El término «centro urbano» permite referirse a cualquier centro, en la medida en que la minería establece vínculos con (o crea) centros de múltiples tallas y funciones, no necesariamente considerados como ciudades.

Sobre la definición de lo que se considera urbano en el Perú, Remy (2013) discute la definición de lo urbano para los censos del INEI que dice que

Área urbana: es la parte del territorio de un distrito cuyas viviendas en número mínimo de 100, se hallan agrupadas contiguamente. Por excepción se consideró urbanas a todas las capitales de distrito.

Las definiciones estadísticas de lo urbano no encajan con lo que normalmente se puede entender como urbano, es decir un cierto dinamismo y una cierta densidad y complejidad en un espacio. Según Vergara, a partir de los diez mil habitantes, un centro poblado empieza a tener una dinámica de atracción migratoria y desarrollo de servicios más complejos (Vergara, 2000 citado por Remy, 2013). El límite entre lo urbano y lo rural no es claro. Una actividad extractiva como la minería puede influir, de distintas formas, en centros poblados de mayor o menor tamaño.

Dicho esto, cuando se habla de centros urbanos mineros, se suele pensar en centros que están directamente ligados a la actividad extractiva, es decir centros que no podrían existir sin una mina. Sin embargo, se considera que es necesario desagregar esta categoría, ya que existen muchos tipos de ciudades y centros que, si bien guardan similitudes entre sí, también pueden tener trayectorias históricas muy distintas.

En un primer momento, se puede clasificar dichos centros en dos grandes categorías. Por un lado, una ciudad minera es aquella que debe su origen y vida a un (o varios) yacimiento(s) y que es, por lo menos en su origen, de función especializada. Sin embargo, podemos también considerar centros de servicios o centros industriales cuyo desarrollo se debe a la comercialización de productos mineros. En general, el inicio de actividades mineras puede o bien crear nuevos asentamientos o bien 
hacer crecer asentamientos ya existentes (Johnston, 1982 citado por Bryceson \& MacKinnon, 2012: 514). Martínez-Fernández et al. (2012: 247) enuncian ciertas características comunes a las ciudades mineras. Estas son la dependencia con respecto a recursos naturales no renovables, la tendencia a localizarse en lugares que se encuentran en la periferia (física o económica) de las áreas metropolitanas, a caracterizarse por una inestabilidad económica y a quedarse fuera de los flujos globales de conocimiento, con un inevitable legado de degradación ambiental. Si bien las características comunes son importantes, hay que recalcar que cada contexto es distinto y las condiciones locales son determinantes en cada caso. Por ejemplo, dependiendo de la configuración de cada sistema urbano en un territorio determinado, la periferia (económica o física) a la que se refieren Martínez-Fernández et al. puede ser entendida de forma distinta.

Desde el punto de vista de los actores que producen estos espacios, se puede distinguir entre centros construidos ad hoc por una empresa o por un Estado para servir a una o varias minas en específico, o centros preexistentes que se ven influenciados por la cercanía relativa de una o varias minas que se empiezan a explotar luego. Muchas veces, los centros ad hoc se han planteado bajo forma de enclave autosuficiente, separado del resto del sistema urbano. A veces este tipo de figura era una necesidad, ya que no existían centros urbanos cercanos que pudieran sostener la vida de una mina remota. Tal es el caso de las ciudades mineras del norte chileno del siglo XIX tales como María Elena, Chuquicamata y Calama, en medio del desierto de Atacama. Esta «constelación de pueblos mineros», como la llama Correa (2016), se desarrolló en función de una red territorial de ciudades y configuró un espacio regional de centros poblados especializados con funciones especificas (vivienda, puerto, comercio) articulados por medio de líneas de ferrocarril.

Ciertos contextos históricos también han dado lugar a centros urbanos que, si bien no son directamente mineros, forman parte de un sistema urbano ligado a la actividad. Por ejemplo, Belo Horizonte, en Brasil, reemplazó a Ouro Preto como capital regional de Minas Gerais hacia el fin del siglo XIX, bajo el impulso de una élite de mineros y cultivadores de café (Correa, 2016). Esta ciudad es un ejemplo del pensamiento de la planificación urbana siguiendo principios higienistas y positivistas europeos (Correa, 2016).

En suma, es necesario remarcar que la categoría de centros urbanos mineros no es homogénea y que los centros se pueden clasificar en distintos tipos siguiendo criterios de función. Los centros pueden ser centros industriales, centros de vivienda, centros administrativos, centros portuarios, centros de intercambio o centros de función mixta. Así, la combinación de varios centros forma un sistema urbano más complejo. Dicho de otra forma, los procesos de extracción, procesamiento y comercialización de minerales pueden darse en lugares distintos; por lo tanto un centro puede servir a la industria minera aún si su función no está ligada a la extracción en sí misma.

De igual forma, los contextos locales e históricos son determinantes en el desarrollo de centros urbanos o regiones ligadas a la actividad minera. 


\section{3. La «maldición de los recursos naturales» y la temporalidad}

Una problemática general ligada a los países que disponen de recursos naturales en abundancia es aquella que se conoce como «maldición de los recursos naturales» o natural resource curse. Significa que, paradójicamente, los países cuyos territorios son ricos en recursos naturales (minerales, petróleo, gas, etc.) suelen tener desempeños económicos malos y niveles de desarrollo bajos (Rosser, 2006: 3). Rosser sostiene que, aún si la evidencia empírica que sostiene esta hipótesis es importante, hay sin embargo varios países que parecen haber logrado convertir esa riqueza en desarrollo (Chile, Botsuana, Indonesia, por ejemplo) pero están lejos de ser la regla y constituyen más bien las excepciones. En el caso de Botsuana, por ejemplo, el Estado logró crear políticas mineras que apuntaron al desarrollo de infraestructura y reducción de la pobreza (Gwebu, 2012: 115) en un contexto de urbanización muy elevada.

En el contexto de la naturaleza cíclica de los booms de commodities, se plantea la pregunta de la capacidad de un Estado (y la inteligencia de los gobiernos) para aprovechar un periodo de alta recaudación fiscal para contribuir a superar la «maldición de los recursos».

Consecuentemente, se plantea la cuestión de la supervivencia de los centros urbanos mineros y del rol que estos pueden jugar en el camino al desarrollo territorial. La temporalidad es entonces, una variable importante. La alta industrialización en Asia ha sido el principal impulsor del súper-ciclo que inició en la década de los años 2000 y que hoy parece estar acabando. Durante este ciclo, el Perú ha visto un importante desarrollo de proyectos mineros formales y minas informales que han tenido impactos en el territorio e influencia en la urbanización.

\section{4. Síntesis: temporalidad y especificidades en la urbanización}

Existen entonces varios puntos que son relevantes en la discusión sobre urbanización y minería.

Un primer punto es sobre la temporalidad finita. Las minas tienen un tiempo de vida limitado que depende de la riqueza de los yacimientos y de su agotamiento. Asimismo, el desarrollo minero está directamente ligado a los mercados globales y a los precios internacionales de los minerales. Esto implica impactos directos sobre espacios locales que se ven afectados por proyectos mineros, en la medida en que una potencial bonanza es siempre pasajera e incierta.

Un segundo punto tiene que ver con el rol del Estado en la administración de esas riquezas naturales y la posibilidad de emplearlas como palanca para el desarrollo. Es decir, la posibilidad de superar la llamada «maldición de los recursos».

Un tercer punto se refiere a la particularidad de cada territorio. Las condiciones locales son muy importantes y con frecuencia, determinantes. En términos geográficos, en América Latina el desarrollo minero suele suceder en zonas alejadas de las grandes ciudades y, por lo tanto, se encuentra más bien asociado a 
ciudades secundarias en zonas predominantemente rurales. La naturaleza y forma de los sistemas urbanos que articulan los territorios en que funciona un sistema minero serán una condición importante en la forma en que dicho sistema se desarrolle. De igual manera, las relaciones entre los actores que producen estos espacios serán condicionantes.

Asimismo, en el marco del proceso actual de urbanización planetaria (Brenner, 2013) es importante observar también los procesos territoriales a varias escalas, particularmente aquellos que son ligados a los actores globales como las compañías mineras multinacionales.

Hay que preguntarse entonces qué tipo de influencia tuvo la minería en los procesos de urbanización, durante los años del ultimo súper ciclo minero en el Perú1. Sin embargo, se propone previamente un pequeño recuento histórico de las relaciones entre sistemas urbanos y minería en el Perú.

\section{TERRITORIOS Y CENTROS MINEROS EN LA HISTORIA DEL PERÚ}

\section{1. Distintos modelos en la historia}

Históricamente, se ha transitado por distintas configuraciones de relaciones de la minería con lo urbano. Se ha pasado de modelos de urbanización directamente ligados a la minería (centros y ciudades coloniales mineros, centros industriales, enclaves o company town, por ejemplo) a otros en que las compañías no producen nuevos espacios urbanos sino que se vinculan con sistemas urbanos preexistentes y aprovechan los elementos del territorio que les pueden ser funcionales.

El régimen colonial español tuvo como pilar fundamental de su economía la explotación de recursos minerales. En consecuencia, la colonización del espacio andino fue esencial para dicho objetivo. Esto dio lugar a la fundación y posterior desarrollo de ciudades en lugares muchas veces inhóspitos, en función de la proximidad a zonas mineralizadas. Según Contreras (1982), la ciudad minera fue «un producto colonial por excelencia: no hubo precedente prehispánico alguno que lo sustentara». Asimismo, Contreras afirma que, dentro de los tipos de ciudades coloniales, la ciudad minera es una que se apartaba del modelo, pues se encontraba generalmente en lugares de difícil acceso y clima, y en ella predominaba un espíritu mercantil y usurero.

Dos casos conocidos son los de la Villa Rica de Oropesa (Huancavelica) y la Villa Imperial de Potosí (Potosí, hoy en Bolivia). Potosí conoció un auge muy importante durante el siglo XVII debido a los yacimientos argentíferos que allí se encontraban. Ambos centros funcionaron como un binomio pues para procesar la plata extraída en el Cerro Rico de Potosí, se utilizaba el insumo de azogue o mercurio que provenía de Huancavelica. Sobre Potosí, Leggett Cahuas (2006: 44) explica que

1 Se estima que el súper ciclo minero en el Perú comenzó en 2002, con la subida de los precios. Este proceso empezó a disminuir en 2014 con la caída de los mismos. 
se trató de una ciudad cuya morfología fue directamente condicionada por la actividad minera que allí se desarrolló: por la estructura social de los habitantes (trabajadores y esclavos indígenas y una élite española en barrios segregados) y por la evolución de las técnicas de extracción de transformación de los minerales, que dieron lugar al desarrollo de infraestructuras de abastecimiento de agua. Sin embargo, otro aspecto importante fue el de la condición regional de Potosí. La ciudad se encuentra en un contexto en que las condiciones naturales volvían muy difícil el desarrollo de otras actividades complementarias (agricultura) que la ciudad necesitaba para existir. En consecuencia, varios otros centros urbanos se desarrollaron con base en actividades productivas o comerciales que permitían el abastecimiento de Potosí. Así se desarrolló una red regional articulada por caminos. Finalmente, la posterior decadencia de Potosí no supuso la decadencia de los demás centros. Por el contrario, como lo dice Leggett:

(...) la centralidad de Potosí (...) supuso una periferia que eventualmente sobrevivió al fracaso de la empresa minera, y que en algunos casos inclusive se benefició directamente de la implosión de la industria argentífera (2006: 48).

De igual forma, Contreras (1982) explica el desarrollo en Huancavelica de un hinterland delimitado aproximadamente entre Pisco, Andahuaylas y Jauja.

Durante el siglo XX, un modelo de minería industrial configuró el espacio de los Andes centrales. Las ciudades de Cerro de Pasco (mina) y La Oroya (refinería) se desarrollaron a partir de la acción de una compañía de capitales norteamericanos, la Cerro de Pasco Copper Corporation (CPCC). Se trató también de un momento de cambio tecnológico: la explotación a cielo/tajo abierto (open pit) reemplazó las técnicas de extracción subterráneas (Vega-Centeno, 2011: 113). El ferrocarril central del Perú, construido hacia el fin del siglo XIX, fue esencial para la articulación de esas ciudades con el puerto del Callao junto a Lima. La región de los Andes centrales sigue siendo la región minera industrial más claramente definida como tal en el Perú. Sin embargo, se trata de una de las regiones más contaminadas, donde los daños al medio ambiente son de los más graves en el mundo. El modelo de producción urbana ligada al desarrollo de esta región fue de hábitat obrero industrial, donde la vida laboral está muy ligada a la vida cotidiana. La actividad agrícola fue reemplazada por la actividad minera. Según Kapsoli (citado por VegaCenteno, 2011), la empresa buscó controlar los circuitos comerciales alrededor de las unidades productivas, lo cual configuró una economía de enclave.

Molotch (1976) concibe las ciudades como «máquinas de crecimiento» impulsadas por los intereses de una élite asentada localmente (Land-based elite, en inglés). En el caso de muchos centros mineros coloniales y republicanos, la analogía es efectiva al punto que, sin aquellos intereses que impulsen dicho crecimiento, difícilmente un centro hubiera siquiera existido en condiciones geográficas tan agrestes como las de los Andes peruanos.

Hoy, si bien aún existen modelos de enclave, así como desarrollos de company town, se observa una tendencia distinta. En los años 1990, las reformas económicas liberales que se pusieron en marcha luego de la crisis económica de los años 1980 
tuvieron como objetivo atraer inversiones extranjeras al Perú. Hoy son muchas las empresas mineras multinacionales que explotan yacimientos a gran escala. Empresas peruanas también participan en proyectos de gran envergadura, muchas veces en asociación con empresas extranjeras. Las nuevas estrategias territoriales de las empresas mineras ya no buscan generar un anclaje en el territorio ni necesariamente producir urbanización. Por el contrario, buscan aprovechar las preexistencias del territorio así como la proximidad relativa de ciertas ciudades que puedan ser funcionales a la mina.

El caso de estudio que nos interesa es el de la ciudad de Huamachuco, La Libertad. Se trata de una ciudad secundaria cerca de la cual se han puesto en funcionamiento dos unidades mineras de minería de gran y mediana escala que han aprovechado la cercanía de la ciudad para satisfacer ciertas necesidades funcionales.

\section{2. Condiciones actuales}

Hoy día, las minas y proyectos mineros en el territorio peruano son numerosos. Existen minas en todas las regiones naturales del Perú (Costa, Sierra y Selva). Sin embargo, la mayor parte de ellas se encuentra en el espacio andino.

Ciertos elementos sientan condiciones para la producción de los nuevos espacios minero-urbanos: las geografías y el conjunto de tres actores principales con sus estrategias y herramientas. A grandes rasgos, los tres actores son el Estado, las compañías mineras y los ciudadanos locales. Cuando hablamos de Estado, es importante diferenciar entre el gobierno central y los gobiernos locales, ya que ambos actúan bajo racionalidades distintas. De igual manera, las compañías mineras no son homogéneas, las hay de distintos tamaños, nacionales y multinacionales, formales, informales e ilegales. Finalmente, los ciudadanos locales o actores sociales son quienes se ven afectados por la presencia minera y reaccionan a dicho estímulo de distintas formas. Los habitantes urbanos parecen ver la presencia minera como una oportunidad económica al mismo tiempo que una amenaza ambiental.

Existen varias herramientas de gestión del territorio y mecanismos de redistribución de los ingresos que genera la minería: el sistema de catastro minero y el de los mecanismos impositivos (canon e impuestos).

Dentro del marco legal peruano, el Estado es propietario del subsuelo. Sin embargo, la superficie no le pertenece necesariamente. Luego de las reformas liberales de apertura de los mercados en los años 1990, las inversiones mineras se multiplicaron. Varias herramientas para facilitar las inversiones se desarrollaron. El sistema de catastro minero (fig. 1) es una de ellas. Este sistema (que es similar a muchos otros en el mundo) está constituido por una grilla imaginaria que define lotes catastrales que se dan en concesión para exploración (y posible explotación) de porciones del subsuelo. En 2014, cerca del 20\% del subsuelo del territorio nacional se encontraba concesionado, mientras que el $1,23 \%$ se encontraba en 


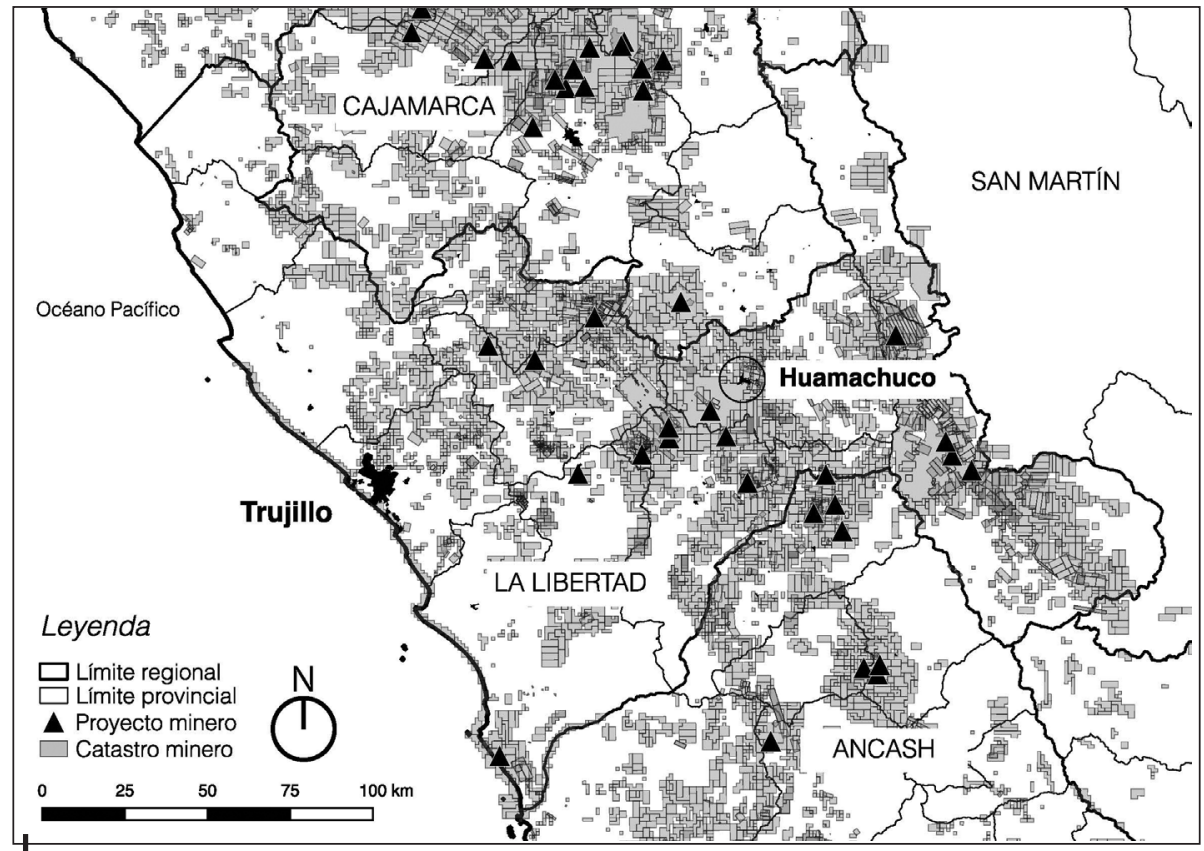

Figura 1 - Concesiones mineras y unidades mineras en la región de La Libertad

Fuente: elaboración del autor con base en datos del GEOCATMIN (sistema de catastro minero)

efectiva exploración o explotación. La región de La Libertad tenía el 61,1\% del subsuelo de su territorio en concesión (Grupo Propuesta Ciudadana, 2014). Se evidencia una visión top-down del Estado que parece ignorar las especificidades de cada territorio. El catastro minero es finalmente una grilla de líneas ortogonales superpuesta a la geografía compleja del país, a partir de la cual se distribuyen los derechos sobre el subsuelo. Estas condiciones alientan lógicas de especulación lo cual trae problemas como superposiciones incompatibles (por ejemplo fuentes de agua y minas) así como una gran incertidumbre sobre el destino de los espacios locales (Grupo Propuesta Ciudadana, 2014). La asignación de lotes catastrales se hace a nivel del gobierno central, en el Ministerio de Energía y Minas MINEM. Muchas veces los actores locales deben sufrir decisiones impuestas desde arriba. Sin embargo, para pasar a la etapa de explotación minera, es necesario que quien la inicie (empresa) sea propietario también de la superficie. Hay entonces negociaciones entre los actores locales (comunidades campesinas, propietarios individuales u otros) y las compañías. Sin embargo, se trata con frecuencia de relaciones de poder asimétricas con desigual acceso a la información. El rol del Estado es constantemente interpelado pues, en las ultimas décadas, los gobiernos parecen haber favorecido siempre los intereses de las empresas en desmedro de los de los ciudadanos afectados. Incluso, ciertas modalidades de la policía nacional permitían que los efectivos prestaran servicios a empresas privadas (mineras) lo cual terminaba por hacer más borrosa la línea entre lo público y lo privado y deslegitimaba el papel del Estado en contextos de conflicto. 
Existen condiciones para una suerte de incertidumbre en relación a los lugares de implantación de un proyecto minero, qué territorios van a verse impactados por él y cómo, lo cual dificulta el planeamiento a largo plazo y pone en duda la capacidad del Perú para superar la «maldición de los recursos».

La tributación minera genera ingresos para el Estado, Ilamados canon. Este es el principal recurso que tienen los gobiernos para producir espacio. El canon minero está definido como «la participación efectiva y adecuada de la que gozan los Gobiernos Regionales y Locales (municipalidades provinciales y distritales) del total de ingresos y rentas obtenidos por el Estado por la explotación económica de los recursos mineros 2$\rangle$. Se trata del $50 \%$ del impuesto a la renta pagado por las empresas que es redistribuido a las regiones, provincias y distritos donde se encuentran las explotaciones mineras, siguiendo ciertos criterios y porcentajes que han variado en el tiempo. El canon debe ser empleado «exclusivamente para el financiamiento o cofinanciamiento de proyectos $u$ obras de impacto regional y local».

Este impuesto ha evolucionado en la historia. En 1976, se trataba de una compensación por extracción de recursos naturales. Luego en 1992, el monto fue establecido en 20\% del impuesto a la renta. En 2001, dicho porcentaje fue elevado a 50\% (Arellano-Yanguas, 2011: 41-44). Los últimos cambios han apuntado a permitir una mejor distribución de los recursos para beneficiar más a las regiones, provincias y distritos en que se ubican las minas. Sin embargo, se encuentran desigualdades: distritos que reciben grandes montos mientras que otros quedan al margen.

El canon minero es el principal medio a través del cual el Estado — particularmente gobiernos locales - actúa sobre el ambiente construido. La mayor parte de las minas se encuentra en territorios predominantemente rurales, en jurisdicciones pobres cuyos exiguos presupuestos se ven súbitamente incrementados por nuevos ingresos que significan oportunidades para desarrollarse. La pregunta es entonces cómo estos impuestos son empleados por los gobiernos para producir el espacio y qué tipo de espacio producen.

\section{CASO DE ESTUDIO: LA CIUDAD DE HUAMACHUCO EN LA PROVINCIA DE SÁNCHEZ CARRIÓN}

La ciudad de Huamachuco (28 330 habitantes en el censo de 2007) se encuentra en el distrito epónimo y es la capital de la provincia de Sánchez Carrión, región de La Libertad, en los Andes del norte del Perú. Huamachuco agrupa funciones y servicios del Estado como administración publica, servicios de salud, educación, etc. La ciudad siempre ha jugado un rol de intermediación y articulación del espacio rural o hinterland con el sistema urbano nacional. Desde una perspectiva de red, Huamachuco es una centralidad que articula centros poblados menores 
así como un hinterland rural. Huamachuco se encuentra bajo la influencia de cuatro explotaciones mineras. De ellas, tres son formales y una es ilegal3:

- Mina La Arena [LA] (operada por Tahoe Resources4) ubicada en la provincia de Sánchez Carrión, distrito de Sanagorán. Es minería formal a tajo abierto que inició operaciones en 2011. Se encuentra a media hora de recorrido de Huamachuco.

- Mina Lagunas Norte [LN] (operada por Barrick Misquichilca) ubicada en la provincia de Santiago de Chuco, distrito de Quiruvilca. Es importante señalar que dicha mina, si bien se encuentra en la jurisdicción de Quiruvilca, está muy cerca de la triple frontera provincial entre Otuzco, Sánchez-Carrión y Santiago de Chuco. Asimismo, se encuentra en la naciente de varias cuencas. Su área de influencia considera las tres provincias. Es minería formal a tajo abierto que inició sus operaciones en 2005. Se encuentra a una hora de recorrido de Huamachuco.

En el Cerro El Toro, visible desde Huamachuco y a quince minutos del centro urbano, operan dos minas:

- Mina El Toro (operada por Corporación del Centro) [CDC] ubicada en la faz sureste del cerro El Toro, a quince minutos de la ciudad de Huamachuco. Es minería formal a tajo abierto. De esta mina disponemos de muy escasa información.

- Minas ilegales en el cerro El Toro ubicadas en la faz noroeste del cerro. Es minería ilegal subterránea.

La situación en El Toro es de convivencia conflictiva. Suelen haber enfrentamientos en la medida en que hay una disputa por el mismo espacio. LN y LA se encuentran ubicadas en la ruta de la carretera que une Trujillo a Huamachuco. El cerro El Toro se encuentra próximo a la ciudad de Huamachuco. La minería en el cerro El Toro existe desde tiempos coloniales, pero se ha intensificado durante los últimos años del súper ciclo. Hoy es notorio el cambio físico del cerro, cuyo volumen poco a poco disminuye.

Los efectos de una mina sobre el territorio tienen varias dimensiones. La primera es aquella ligada directamente a la explotación. LN se encuentra en el mismo lugar que varias nacientes de cuencas y, por lo tanto, los impactos son sobre varios

3 El Estado peruano clasifica las minas siguiendo dos criterios: el tamaño de la explotación y el estatus de formalidad. Según el tamaño, las escalas en minería son grande, mediana, pequeña y artesanal. Esto se hace con relación en la capacidad productiva de las minas. Según el estatus de formalidad, las minas son consideradas como formales o no formales. Las formales son aquellas que operan dentro del marco legal. Las no formales se dividen en informales e ilegales. Las primeras son las que se encuentran en proceso de formalización mientras que las segundas se encuentran fuera de la legalidad y son combatidas. Una correlación bastante evidente es que las minas de mayor escala son formales mientras que las de escala menor tienden a la no formalidad. Ciertamente, muchas veces los límites de la formalidad son borrosos.

4 Hasta 2015, se trataba de la empresa Rio Alto Mining Ltd. En abril de 2015 ambas empresas se fusionaron. 
cursos de agua. LA se encuentra aguas abajo en una de esas cuencas. Las minas a cielo abierto requieren gran cantidad de espacio físico, enormes cantidades de tierra son desplazadas y el paisaje es fuertemente modificado. LN y LA se encuentran alejadas de la ciudad de Huamachuco, pero mantienen vínculos funcionales con ella. LA, al estar en la jurisdicción de Sánchez Carrión y más cerca de Huamachuco que LN, se vincula de manera más directa. La segunda dimensión es aquella de los efectos en los centros urbanos cercanos. Desde el inicio de las operaciones de todas estas minas, se han sucedido diversos cambios urbanos a varias escalas. Estas transformaciones resultan de las interacciones entre las empresas mineras, el gobierno local y la población local y se dan a distintas escalas del territorio. Se plantea un análisis de cambios en el espacio a dos escalas: la de la ciudad de Huamachuco y la de la provincia de Sánchez Carrión, de la cual Huamachuco es capital. Se evoca una escala adicional: la de la relación entre Huamachuco y Trujillo.

\section{1. Regalías y canon: medios de producción espacial a escala provincial}

Todo proyecto minero tiene áreas de influencia directa y áreas de influencia indirecta. Ellas son determinadas por los Estudios de Impacto Ambiental (EIA) que las empresas formulan y el Estado aprueba. Estas áreas están habitadas y por lo tanto sufren ciertos impactos. Se trata de espacios rurales ocupados por centros poblados dispersos. Esto ha resultado en la creación de ciertos espacios de diálogo entre las autoridades de las tres provincias y la empresa. Por ejemplo, el proceso de negociación entre LN y los gobiernos de las tres provincias que se encuentran en el área de influencia de la mina ha dado como resultado la creación de una asociación civil Ilamada Fondo Social Alto Chicama (FSAC), creada en 2009, cuya misión es ejecutar los fondos provenientes de las regalías aportadas por Barrick. Dichos fondos se emplean en construcción y refacción de infraestructura así como en programas sociales puntuales (educativos y otros). Los proyectos son decididos de manera concertada entre autoridades de las tres provincias, de los tres distritos y representantes de la empresa minera $\mathrm{LN}$.

Si nos enfocamos en las obras de infraestructura que se han ejecutado, vemos que, desde un punto de vista espacial, las acciones en el ambiente construido ejecutadas por el FSAC pueden ser clasificadas en dos tipos: lineales (vías, canales de irrigación, etc.) y puntuales (edificios, escuelas, postas médicas, etc.). Geográficamente, dichas obras se distribuyen de manera atomizada en el territorio de las tres provincias. La construcción de dichas obras responde pues a demandas locales de los caseríos o centros poblados. Solamente las capitales provinciales (Otuzco, Santiago de Chuco y Huamachuco) concentran obras más significativas. Sin embargo, otras acciones estructuradoras del territorio existen. La carretera que une Trujillo y Huamachuco, por mucho tiempo en mal estado, fue reparada siguiendo un acuerdo entre el Ministerio de Transportes y Barrick. Esto nos habla de cómo la presencia e influencia de una mina puede movilizar elementos del 
territorio que les son funcionales y que a la vez son importantes para el territorio en el que se inserta dicha mina.

A la escala de la provincia de Sánchez Carrión, analizamos también la ejecución de obras de infraestructura de la municipalidad entre los años 2007 y 2014 (figs. 2 y 3). En el caso de la MPSC, el canon minero ha sido una de las fuentes de financiamiento más importantes. Se trata en general de obras relativamente pequeñas, aunque a veces significativas. Puentes, mejoramiento de vías locales, construcción o refacción de locales comunitarios, escuelas, etc. En términos espaciales, vemos que las obras tienden a concentrarse en la ciudad de Huamachuco y en los centros poblados o caseríos cercanos. Como se mencionó anteriormente, en términos del tipo de red urbana, Huamachuco es una centralidad que articula centros menores. En ese sentido, parece lógica la tendencia a consolidar el centro del sistema.

\section{2. La ciudad de Huamachuco: un lugar de acciones superpuestas}

En la ciudad de Huamachuco, los cambios más evidentes están en la morfología urbana. En este espacio se superponen la acción pública y la privada. La ciudad es, según Remy \& Voyé (2006), el «lugar en que las interrelaciones son decisivas y se traducen en la morfología [urbana] misma».

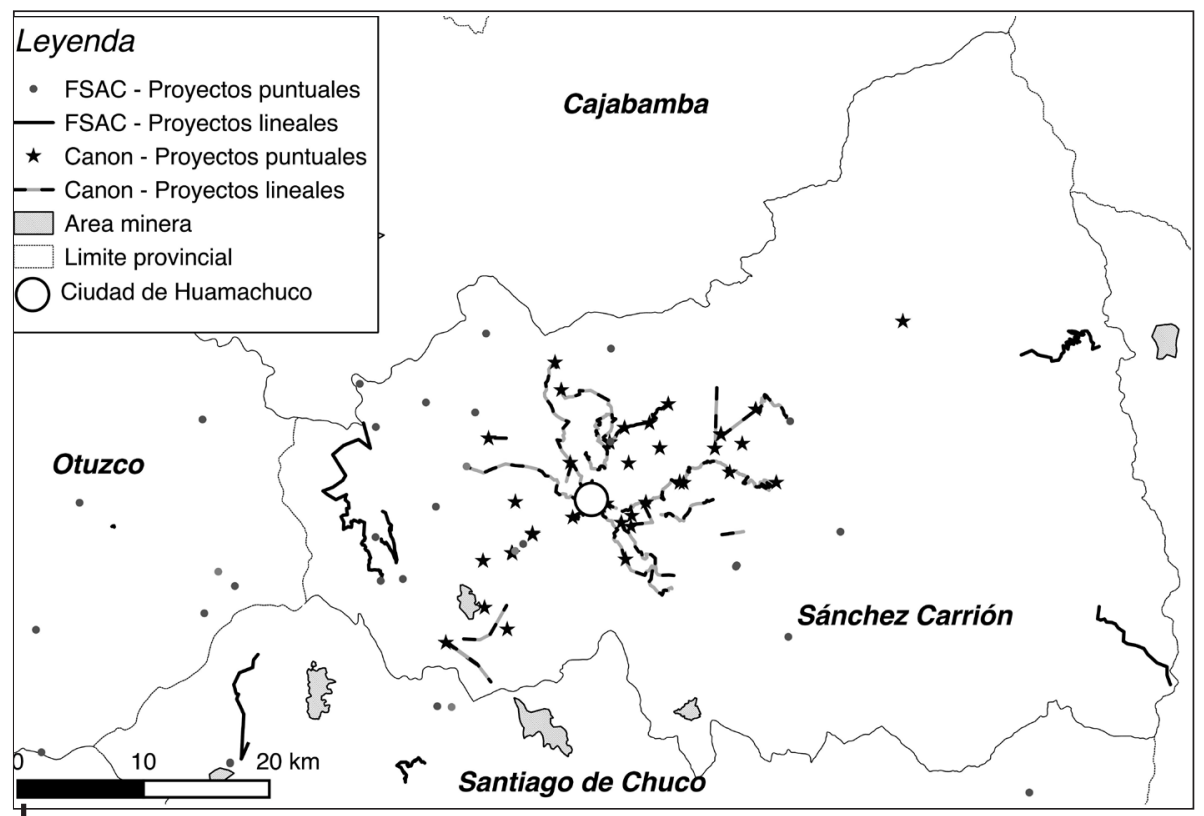

Figura 2 - Provincia de Sánchez Carrión. Obras proyectadas por la municipalidad provincial de Sánchez Carrión MPSC entre 2007 y 2014. Obras proyectadas por el Fondo Social Alto Chicama (FSAC) entre 2009 y 2014

Fuente: elaboración del autor con base en datos del FSAC y de la MPSC 


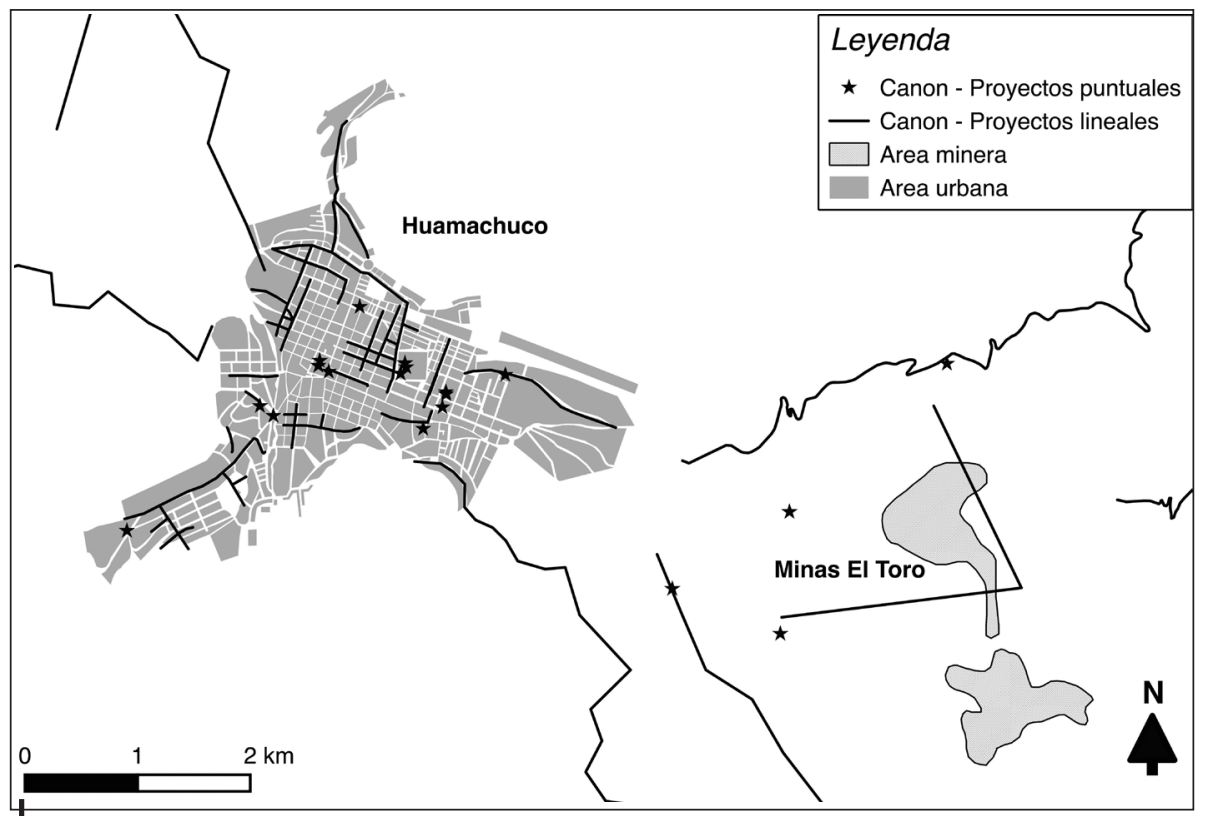

Figura 3 - Ciudad de Huamachuco, minas de El Toro y proyectos municipales financiados con canon minero

Fuente: elaboración propia con base en datos de la Municipalidad Provincial de Sánchez Carrión

Huamachuco se volvió en parte funcional a las necesidades de las minas LN y LA. Las compañías, especialmente LA, buscan aprovechar la proximidad relativa de la ciudad para satisfacer las necesidades de alojamiento y alimentación de sus trabajadores. La demanda de servicios generada por la mina hizo reaccionar a los actores locales y diversos encadenamientos económicos se produjeron. Nuevos edificios con funciones nuevas (hoteles, restaurantes, comercio) se han construido y modifican las formas y dinámicas de la ciudad (fig. 4). Paralelamente, la ciudad se ha vuelto atractiva ya que se perciben posibilidades económicas. Tiendas de todo tipo se multiplican y la ciudad se expande tanto horizontalmente hacia las periferias, como verticalmente en las áreas centrales (Bensús \& Pérez, 2014: 32). Sin embargo, esta rápida densificación constructiva no es sin presentar problema. Por ejemplo, los hoteles que antes estaban ocupados (durante la etapa de implementación de las minas) hoy se encuentran a mitad vacíos. Esto se debe al hecho que la demanda de alojamiento bajó cuando las minas (que operan de manera tecnificada) pasaron de la etapa de implementación a la etapa de operación. De igual forma, la expansión urbana se da sobre zonas de riesgo como laderas de cerros y áreas naturales, como los humedales de Purrumpampa. Vemos pues problemáticas ligadas a la temporalidad de la influencia minera que estimula la economía urbana local de manera veloz, estímulo y crecimiento que al gobierno local le cuesta organizar. En entrevistas con funcionarios del área 


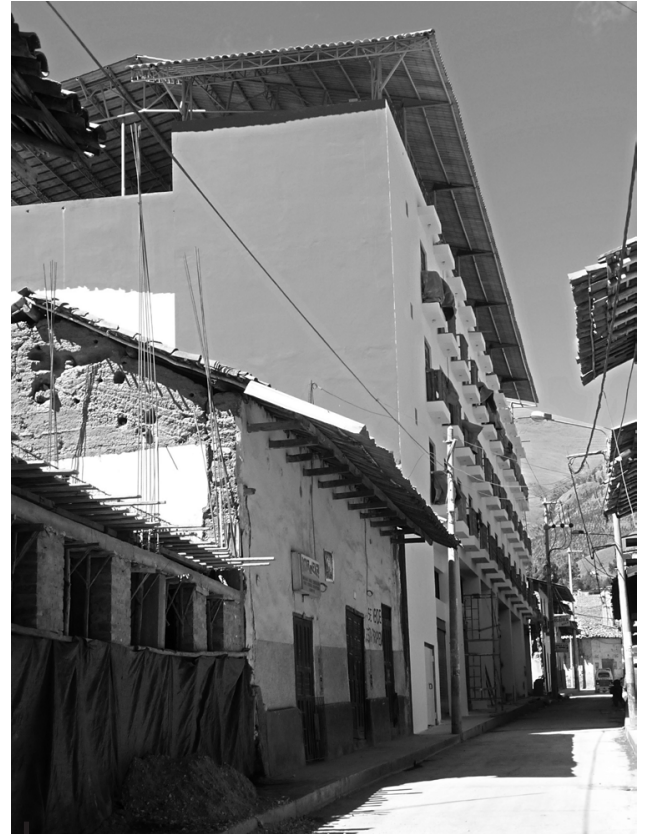

Figura 4 - Huamachuco. Hotel nuevo en el tejido urbano preexistente

(C) Alejandro Gonzales Gavilano

de desarrollo urbano de la MPSC, se pone en evidencia la escasa capacidad de control de las nuevas edificaciones.

Por otro lado, mecanismos de tributación nuevos como las Obras por Impuestos [OXI] han permitido también una acción directa de las empresas mineras en el espacio urbano. Recientemente, se ha remodelado la Plaza de Armas a través de este mecanismo y se encuentra en ejecución un proyecto de remodelación del mercado municipal, ambos por la compañía LA.

\section{3. La minería en El Toro y sus caseríos}

Las minas LA y LN que se encuentran físicamente alejadas de Huamachuco influyen tanto en la ciudad como en la región, aunque de manera más bien indirecta. Sin embargo, la minería informal presente en el cerro El Toro tiene un correlato directo con el crecimiento y la consolidación de ciertos espacios urbanos.

En primer lugar, los centros menores Shiracmaca y El Toro son dos caseríos que se encuentran en las faldas del cerro El Toro y que han crecido a raíz de la minería. Sus habitantes se han enriquecido. Datos del censo de 2007 muestran que Shiracmaca tiene porcentajes importantes de población migrante y de población que consigna la minería como ocupación principal. El porcentaje de población migrante en 2007 en Shiracmaca era de 14,41\% (INEI, 2007), más alto que el resto de caseríos aledaños a la ciudad. Paralelamente, se observa que en el material de construcción de viviendas, existe un mayor porcentaje de ladrillo como material de paredes con relación en otros caseríos cercanos a Huamachuco. Es decir que estos caseríos parecen haberse consolidado físicamente y adquirido una especificidad en cuanto a sus actividades económicas. Es interesante notar que se puede ver la acción de la MPSC en estos lugares. Entre las obras viales, cuyo financiamiento se consigna como canon minero, se encuentra el mejoramiento de vías de acceso al cerro El Toro, por el norte y por el sur. Parece paradójico que la acción pública ayude a consolidar una accesibilidad que, si bien beneficia a los habitantes, también puede ayudar al desarrollo de la minería ilegal que el Estado debe, en principio, combatir. De alguna manera, se termina legitimando la minería ilegal a través de estas obras.

En segundo lugar, la minería informal parece tener mayores vínculos con Huamachuco que la minería formal. En una entrevista, un minero de El Toro indicó que había comprado propiedades en Huamachuco y en Trujillo. Asimismo, Bensús \& Pérez (2014: 39) indican que tanto los patrones como los peones de 
la minería ilegal «ven a Huamachuco como un lugar en el cual establecerse, en especial porque para muchos de los trabajadores — quienes se dedicaban a la agricultura - llegar a la ciudad supone una mejora real y simbólica en su calidad de vida». Damonte (2016) argumenta que, en relación con el boom minero en el Perú, se ha producido un desarrollo de los espacios urbano-rurales de residencia. Dice Damonte:

La mejora de la infraestructura y los servicios públicos y privados — como el transporte y la educación- ha determinado que muchas familias campesinas busquen mantener un espacio en ciudades intermedias del ámbito rural. Así, la ciudad se vuelve un espacio vivencial y de aprendizaje para los miembros de las familias campesinas, quienes integran la experiencia urbana en su proceso de socialización, al tiempo que se apropian de estos espacios (2016: 31).

El caso de Huamachuco parece corresponder a esta lógica. Sin embargo, es la presencia de la minería del cerro El Toro la que parece motivar una mayor permanencia de las personas en el espacio urbano. Esto se debería a la proximidad del cerro El Toro con la ciudad de Huamachuco.

\section{SÍNTESIS Y CONCLUSIONES}

Hemos visto que las actividades mineras han jugado un rol fundamental en la estructuración histórica del territorio peruano, particularmente del espacio andino. Sin embargo, la naturaleza de esa estructuración ha cambiado a lo largo de la historia. En un primer tiempo, fue una estructuración directa a través de las ciudades coloniales ligadas directa o indirectamente a las minas. Luego, se desarrollaron por regiones mineras en el siglo XX, en una lógica más industrial con una producción urbana ligada a modelos de company town. Hoy, la naturaleza de las minas (y de los actores que las operan) es distinta y también lo es la relación con el territorio.

Las compañías multinacionales, que hoy funcionan con tecnologías intensivas en capital y que están ligadas a flujos globales, tienen estrategias territoriales nuevas que apuntan a aprovechar las preexistencias y a crear relativamente pocos vínculos con los espacios locales. La producción espacial y urbana que deviene de la minería se ha convertido en indirecta. Sin embargo, la minería informal o ilegal, al ser menos intensiva en capital, parece tener un anclaje mayor en el territorio en que existe, en la medida en que estos mineros son gentes con arraigo local.

La influencia minera se constata a todas las escalas del territorio. El espacio se transforma, ya sea a través del canon minero, de las regalías, por la acción directa de las empresas, por la del Estado o por la de la población local. Molotch (1976) argumentaba que una ciudad es una «máquina de crecimiento» cuyo móvil son los intereses de una élite local basada en la tierra. En este caso, cabe preguntarse si en el caso de Huamachuco hay una élite que impulsa este crecimiento de la máquina. Parece ser que una nueva élite económica local de mineros ilegales efectivamente impulsa el crecimiento tanto del espacio local como de otros 
centros (Trujillo). Al mismo tiempo que actores supranacionales, como las mineras Barrick y Tahoe, generan estímulos (más indirectos) que provocan una mutación de la ciudad y de la región.

Dentro de los distintos tipos de centros urbanos mineros o vinculados a la minería, el caso de Huamachuco y su región es el de un centro preexistente que se ve influenciado por los distintos tipos y escalas de minería. Retomando el concepto de «urbanización mineralizada», podemos ver que, en relación con la posición de Huamachuco en el sistema urbano mayor, su carácter de centralidad secundaria de la región se ha reforzado al haber atraído a migrantes y desarrollado cierto nivel de especialización económica.

Es importante notar que la manera en que la influencia minera ha ocurrido tiene ciertas condicionantes relevantes que pueden agregar un factor de aleatoriedad. El sistema de catastro minero es un sistema que nada tiene que ver con unidades funcionales del territorio como cuencas o divisiones políticas y por lo tanto es un elemento de incertidumbre sobre el uso del subsuelo y suelo. Por ejemplo, la ubicación física de la mina Lagunas Norte, en una triple frontera administrativa y entre múltiples nacientes, indujo una estrategia territorial particular de relacionamiento de la compañía Barrick con el entorno (la creación del FSAC). Esa relación posiblemente hubiera sido otra si la mina se hubiera ubicado en otro punto de la concesión. En otras palabras, se trata de varios sistemas que se sobreponen en el mismo espacio pero que no necesariamente se conectan: el sistema urbano, el sistema minero, los actores que los activan.

El caso de la región de La Libertad y la ciudad de Huamachuco nos ha permitido ilustrar los efectos que la actividad minera tiene sobre un territorio específico. La producción territorial hecha por los distintos actores (Estado, empresas, población) no sigue una visión común. Las unidades mineras parecen adaptar elementos del territorio a sus necesidades. Surge la pregunta de cuáles serían las condiciones para hacer que suceda lo contrario, es decir que sean las necesidades de esos territorios las que permitan imponer las condiciones de integración a las minas. Finalmente, cabe preguntarse cuál ha de ser el futuro de Huamachuco en un escenario posminas. Una visión de desarrollo territorial puede ser una clave importante para planear este futuro y, en un escenario más amplio, contribuir a superar la «maldición de los recursos».

\section{Referencias citadas}

ARELLANO YANGUAS, J., 2011 - ¿Minería sin fronteras? Conflicto y desarrollo en regiones mineras del Perú, 324 pp.; Lima: IEP, PUCP, Universidad Antonio Ruiz de Montoya.

BRENNER, N., 2013 - Tesis sobre la urbanización planetaria. Nueva Sociedad, 243 (enerofebrero): 38-66.

BRYCESON, D. \& MACKINNON, D., 2012 - Eureka and beyond: mining's impact on African urbanisation. Journal of contemporary african studies, 30 (4): 513-537. 
BENSÚS, V. \& PÉREZ, A., 2014 - Nuevas dinámicas territoriales en ciudades intermedias. El caso de Huamachuco, La Libertad, 73 pp.; Lima : Pontificia Universidad Católica del Perú. Cuadernos Arquitectura y Ciudad, 20.

CONTRERAS, C., 1982 - La ciudad del mercurio. Huancavelica, 1570-1700, 126 pp.; Lima: IEP Ediciones.

CORREA, F., 2016 - Beyond the City: Resource Extraction Urbanism in South America, x + 166 pp.; Austin: University of Texas Press. doi:10.7560/309414

DAMONTE, G., 2016 - Transformación de la representatividad política local en los contextos extractivos a gran escala en los Andes peruanos. In: Industrias extractivas y desarrollo rural territorial en los Andes peruanos: los dilemas de la representación política y la capacidad de la gestión para la descentralización (G. Damonte \& M. Glave, eds.):19-58; Lima: Grupo de análisis para el desarrollo (GRADE).

GRUPO PROPUESTA CIUDADANA, 2014 - Concesiones mineras en el Perú. Análisis y propuestas de política, 91 pp.; Lima : Propuesta Ciudadana.

GWEBU, T. D., 2012 - Botswana's mining path to urbanisation and poverty alleviation. Journal of Contemporary African Studies, 30 (4): 611-630.

INSTITUTO NACIONAL DE ESTADÍSTICA E INFORMÁTICA (INEI), 2007 - Censos Nacionales : XI de Población y VI de Vivienda; Lima.

KOSTOF, S., 1991 - The City Shaped. Urban patterns and meanings through history, 352 pp.; Londres: Thames and Hudson.

LEGGETT CAHUAS, G., 2006 - Industria, crisis y urbanismo en la Villa Colonial de Potosí (siglos XVI-XVII). ur[b]es, 3: 37-52.

MARTÍNEZ-FERNÁNDEZ, C., TONG WU, C., SCHATZ, L. K., TAIRA, N., \& VARGAS HERNÁNDEZ, J. G., 2012 -The Shrinking Mining City: Urban Dynamics and Contested Territory. International Journal of Urban and Regional Research, 36 (2): 245-260.

MOLOTCH, H., 1976 - The city as a Growth Machine: Toward a Political Economy of Place. The American Journal of Sociology, 82 (2): 309-332.

REMY, M. I., 2013 - Reflexiones sobre lo rural (y lo urbano) en el Perú. In: Ciudades intermedias y desarrollo territorial ( J. Canziani \& A. Schejtman, eds.): 71-82; Lima: Fondo Editorial de la Pontificia Universidad Católica del Perú.

REMY, J. \& VOYÉ, L., 2006 - La ciudad: ¿Hacia una nueva definición?, 314 pp.; Zaragoza: Bassarai Ediciones.

ROSSER, A., 2006 - The Political economy of the Resource Curse: a Literature Survey. Working paper series, 268; Brighton: IDS.

VEGA-CENTENO, P., 2011 - Los efectos urbanos de la minería en el Perú: del modelo de Cerro de Pasco y La Oroya al de Cajamarca. Apuntes: Revista de Ciencias Sociales, 38 (68): 109-136. 